\title{
Diffusion des Wasserdampfs auf der Oberflächenschicht der Schneedecke
}

\author{
von \\ Eiji Uchida \\ Meteorological Research Institute, Tokyo \\ (Recieved Octorber 31, 1960)
}

\begin{abstract}
Zusammenfassung
Die Profile von Windgeschwindigkeit, Temperatur und Feuchtigkeit (Taupunkt) wurden auf den Oberflächen der Schneedecke und der mit Wasser angefeuchteten Gaze gemessen.

Wenn die Windgeschwindigkeit vergleichsweise klein ist, kann die Dicke der Laminarsubschicht (und daraus auch der Fluss der Wasserdampfs) vermutet werden.

Diese Methode ist, scheint es, einfacher in dem Prinzip und besser in der Genauigkeit als die Methode vom sogenannten Logarithmen-Gesetz in der Turbulenzschicht.

Dabei wurden die Replicas in der Oberflächenschicht der Schneedecke im Zusammenhang mit dem Fluss des Wasserdampfs untergesucht.
\end{abstract}

\section{Einleitung}

Seit 1958 sind die Beobachtungen des Flusses des Wasserdampfs in der Oberflächenschicht der Schneedecke im Winter zu Kumanoyu (in Shiga Plateau) fortgesetzt worden, aber die feinen Resultate konnten wegen der fraglichen Genauigkeit des Instrumentes und der Formeln des Flusses nicht gewonnen werden.

Im Februar 1958 wurde das kleine Thermistor-Psychrometer nach oben und unten bewegt, und der Fluss wurde aus der Date der Profile der Feuchtigkeit berechnet. Die wärmefühlenden Teile des Psychrometers sind zwei feinen Thermistoren $(0.6 \sim 0.9 \mathrm{~mm} \phi$, $4 \mathrm{~mm}$ Länge, 10 sek Zeit-Konstante), von welchen das eine feuchte Thermistor ist mit feinen, angefeuchteten Seidenfaden gebunden, und die Distanz zwischen den feuchten und trocknen Thermistoren ist $2 \mathrm{~cm}$.

Im Laboratorium zeigt dieses Psychrometer eine Übereinstimmung mit dem AssmanPsychrometer in Genauigkeit von $\pm 0.1^{\circ} \mathrm{C}$.

Noch dazu, die Profile von Windgeschwindigkeit und Temperatur wurden mit einem Thermistor-Anemometer (Kugelform, $1 \mathrm{~mm} \phi, 2$ sek Zeit-Konstante), einem WärmelinieAnemometer (Platinlinie, $10 \mu \phi, 2 \mathrm{~mm}$ Länge, 0.01 sek Zeit-Konstante) und einem Thermistor-Thermometer (Kugelform, $1 \mathrm{~mm} \phi$, 14 sek Zeit-Konstante) gemessen (Höhe, 0.5 $100 \mathrm{~cm}$ ). Als Formeln des Flusses, wurden dieselben von Monin und Obukhov (1954) gebraucht. Diese sollen eigentlich für ein Luftgebiet von etwa $50 \mathrm{~cm} \sim 10 \mathrm{~m}$ Höhe gebraucht werden, aber es wurde vermutet, dass sie noch bis $20 \sim 30 \mathrm{~cm}$ unter der Schneeoberfä̈che gültig sein mag. Daraus wurde der Fluss in der Nacht $\left(V ; 2 \mathrm{~m} / \mathrm{s}, T ;-15^{\circ} \mathrm{C}\right)$ als $-0.5 \sim-2.0 \mathrm{gr} / \mathrm{m}^{2} \mathrm{~min}$ berechnet. (Negatives Zeichen zeigt Verdampfung.) 
Im März 1959 wurden die Wärmegefühlsteilen ins Quecksilver in einem Glasrohr $(0.5 \mathrm{~cm} \phi, 3 \mathrm{~cm}$ Länge, Zeit-Konstante: um etwa 1 min verlängert, um den Durchschnittswert zu gewinnen) eingetaucht (Höhe: $10 \sim 200 \mathrm{~cm}$ ).

Dabei wurde, ums Diffusion-Koeffizient aus der praktischen Messung zu berechnen, der Rauch von $\mathrm{Ticl}_{4}$ in verschiedenen Höhen strömen lassen, und die Diffusionskoeffizienten konnten aus den Diffusionswinkeln berechnet werden. Der Fluss war $-1.1 \mathrm{~g} / \mathrm{m}^{2} \mathrm{~min}$ in Abend $\left(V ; 6 \mathrm{~m} / \mathrm{s}, T ;-4^{\circ} \mathrm{C}\right)$ aus den Profile und den Diffusionskoeffizienten.

Aber hier handelt es sich um die Genauigkeit von dem Instrument, von den Formeln

des Flusses, von den Werten der Diffusion-Koeffiziente usw, da die Ergebnisse uns nicht immer befriedigen wird. Zuerst, die Formeln des Flusses sind im Gebiet der Turbulenz nicht bestätigt, und im wichtigsten Gebiet neben der Grenzfläche können sie infolge der Einwirkung der Molekularviskosität nicht gebraucht werden.

Dann wäre es nicht besser, dass die Messung des Gradienten der Feuchtigkeit (Taupunkt) in der dünnen Laminarschicht der Grenzfläche (Laminarsubschicht) gemacht werden soll? Leider aber, nach dem gesunden Menschenverstande wird diese Schicht von mehreren Ursachen wie Windgeschwindigkeit und Rauheit dünner werden. Aber wenn irgend ein Grenz (Windgeschwindigkeit von einigen $\mathrm{m} / \mathrm{s}$, sage etwa $5 \mathrm{~m} / \mathrm{s}$, Rauheit wie auf der Schnee-oder Wasseroberfläche, Temperatur über etwa $-15^{\circ} \mathrm{C}$, für die Genauigkeit der Taupunktmessungen gegeben würde), so wäre diese Messung möglich. Dann wird die Theorie der Verdampfung sehr einfach, und der Wert des Flusses wird mit besserer Genauigkeit gerechnet.

\section{Instrument der Beobachtung}

Im März 1960, als Beobachtungsinstrumente wurden ein Thermistor-Anemometer

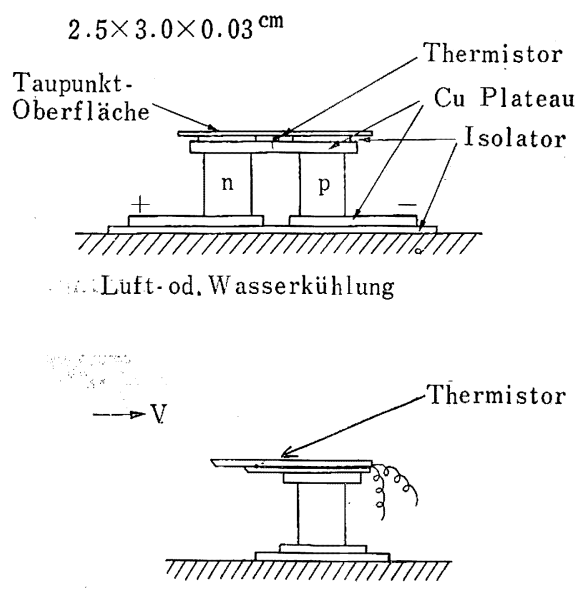

Luft-od. Wasserkithlung

Abb. 1. Elektrische Taupunktmessungen. (Thermoelektrischer Refrigerator)

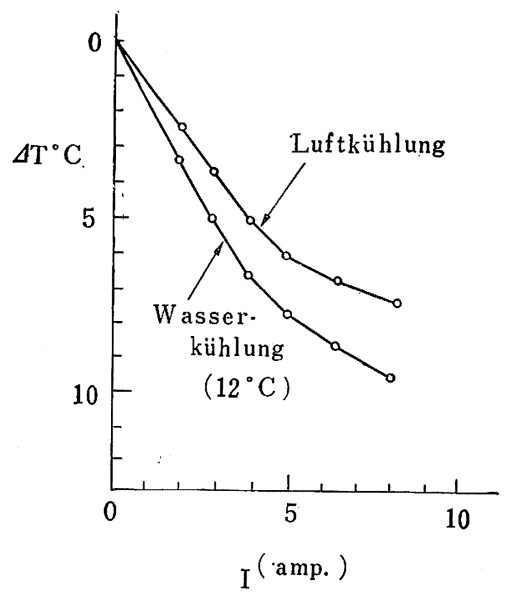

Abb. 2. Die Kurve des Temperaturfallens $\left(\Delta T=T_{1}-T\right)$. $\left(T_{1}\right.$ : Zimmertemperatur, $T$ : Temperatur auf der Taupunktfläche) gegen elektrische Strömung (I) 
$(0.5 \mathrm{~mm} \phi)$, ein Thermistor-Thermometer $(0.1 \mathrm{~mm} \phi)$ und ein elektrisches Taupunktmeter gebraucht. Unter den jetzigen Zuständen ist das Taupunktmeter vergleichsweise in besserer Genauigkeit, bzw, es ist nützlich für die Fälle des Unterschieds zwischen Tau und Reif, der Unnützlichkeit des Wasserangebots, der Genauigkeit gegen die Höhe, und der Berechnung des Flusses aus dem Wassererdampfdruck usw. Als Kühlapparat wurde das Thermoelement (ein thermo-elektrischer Refrigerator) gebraucht (Abb. 1 Photo, 1). Wenn der elektrische Gleichstrom (I) von einem Semikonduktor von n-Typus nach demjenigen von p-Typus geströmt wird, wird die Temperaturdifferenz $z$ wischen den oberen und unteren Pol-Flächen erzeugt, und die Temperatur der oberen Fläche ( $\mathrm{T})$ wird tiefer als die Zimmertemperatur $\left(T_{1}\right)$, wenn die untere Fläche mit Luft oder Wasser abgekühlt wird. Die Taupunktfläche ist Chrom-Kupferplatte $(2.5 \times 3.0 \times 0.03 \mathrm{~cm})$ plattiert, und von der refrigerierten Fläche elektrisch abgeschnitten, und an ihrer inneren Seite mit einem ThermistorThermometer $(0.1 \mathrm{~mm} \phi)$ angeklebt.

Die Kurve $\measuredangle T$ gegen I (wo $\Delta T=T_{1}$ (Zimmertemp.) $-T$ (Temp. auf der Taupunktfläche, und $\mathrm{I}=$ elektrische Strömmung) ist in Abb. 2 dargestellt (Wassertemp.: $12^{\circ} \mathrm{C}$ ). Im Freien wurde die Luftkühlung gebraucht. Die Temperatur des Zimmers von konstanter Feuchtigkeit wurde durch die schwefelsäure Lösung in der Genauigkeit von $\pm 0.1^{\circ} \mathrm{C}$ reguliert behalten.

Es kommt auf die Refrigerierungsgeschwindigkeit unter $0.25^{\circ} \mathrm{C} / \mathrm{min}$ und die Windgeschwindigkeit unter etwa $5 \mathrm{~m} / \mathrm{s}$ an, wenn man den richtigen Wert des Taupunktes gewinner will. (Zeit-Konstante ist 69 sek in der Luftkühlung.) Die Temperaturdifferenz zwischen dem gefallenen Tau und dem getrockneten ist $\pm 0.1^{\circ} \mathrm{C}$ mit $0.25^{\circ} \mathrm{C} / \mathrm{min}$.

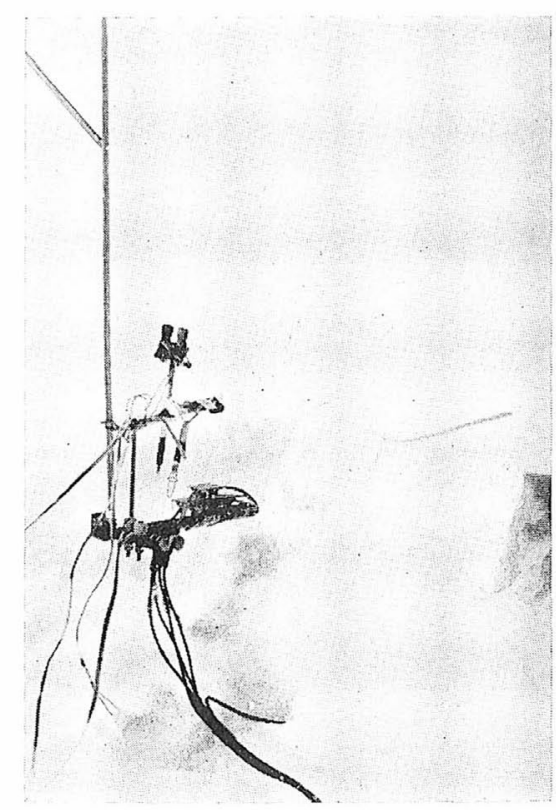

Photo 1. Taupunktmessung.

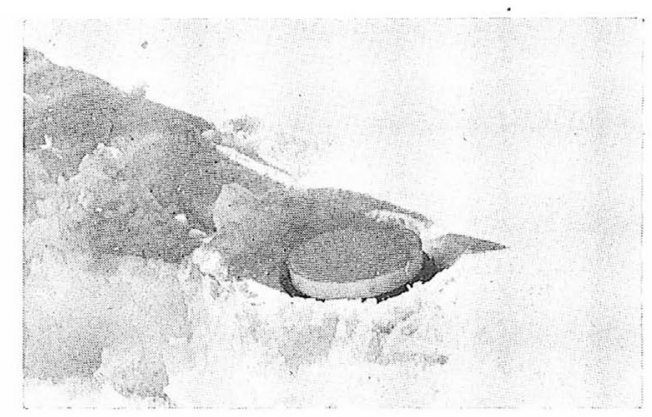

Photo 2. Verdampfungsmessung.

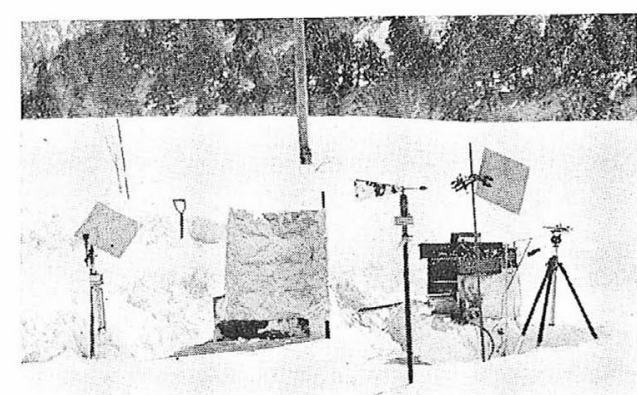

Photo 3. Zusammengesetzte Beobachtungen. 


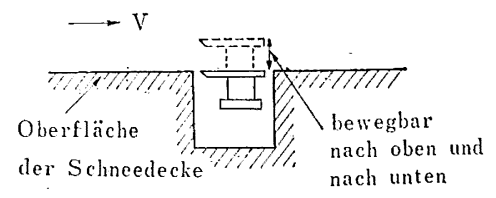

Abb 3. Messung des Taupunktes.

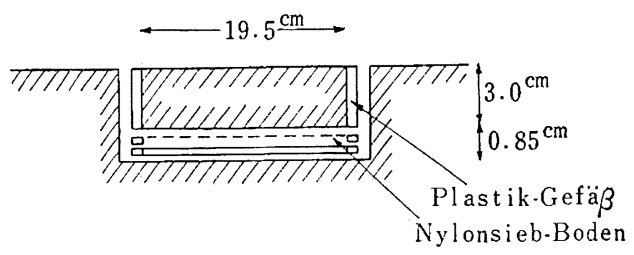

Abb. 4. Verdampfungsmessungen.
Im Falle der Beobachtung wurde die Schneedecke wie in Abb. 3 abgeschnitten, und natürlich, die Sonnenstrahlen ging auf die Elementen entgegen.

Bei den Verdampfungsmessungen wurde ein plastisches Gefäss $(19.5 \mathrm{~cm} \phi, 3.0 \mathrm{~cm}$ Tiefe) vorbereitet. Ihm war der Boden abgestossen und ein Sieb von Nylon dafür von der Querseits aus gesteckt, und das Gafäss wurde in die natürlich Schneedecke hineingesteckt. Abb. 4, Photo 2 zeigt die abgeschnitten Formen (Genauigkeit $\pm 0.2 \mathrm{gr}$ in der $\mathrm{Me}$ ssungen von der Waage).

\section{Beobachtung und Ergebnisse}

Am 19. März 1960, in Hiratoko Ebene, Kumanoyu (Shiga Plateau), wurden die zusammenfassenden Beobachtungen gemacht (Photo 3). Die Übersicht von ihnen ist in Abb. 5 und 6. dargestellt. Windgeschwindigkeit und Windrichtung waren vergleichsweise stabil,mit Ausnahme von den Richtungsabwendungen am Abend. Es gab keineWolken, und auch das Sammeln der Replicas von der Schneedecke wurde gemacht.

Die Data von der Dichtigkeit der Schneedecke und von der Temperaturverbreitung in der Schneedecke (mit Thermistor-Thermometer gemessen, Werte des einigemaligen Durchschn-

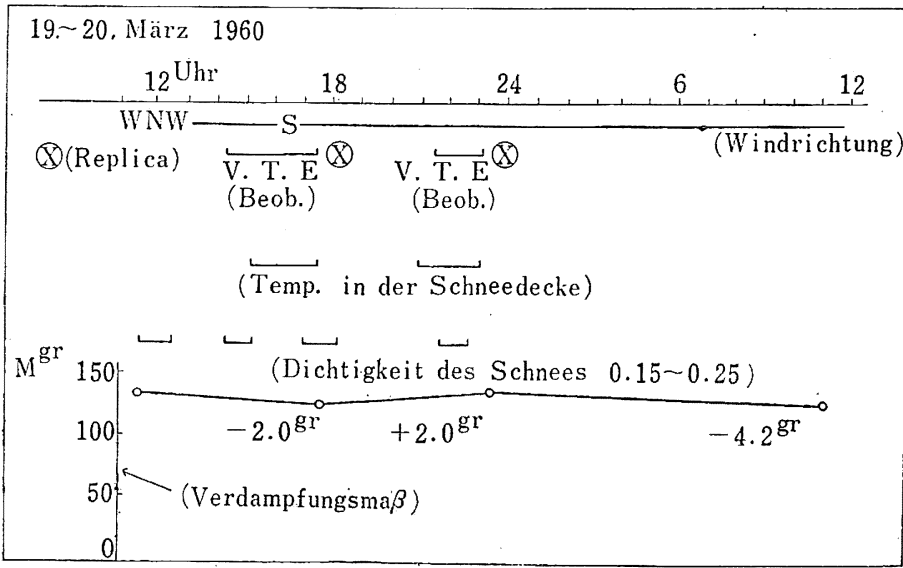

Abb. 5. Übersicht der Beobachtungen zu Hiratoko Ebene, Kumanoyu (Shiga Plateau). (V: Windgeschwindigkeit, T: Temperatur, P: Wasserdampfdruck; M: Verdampfungsmasse) 


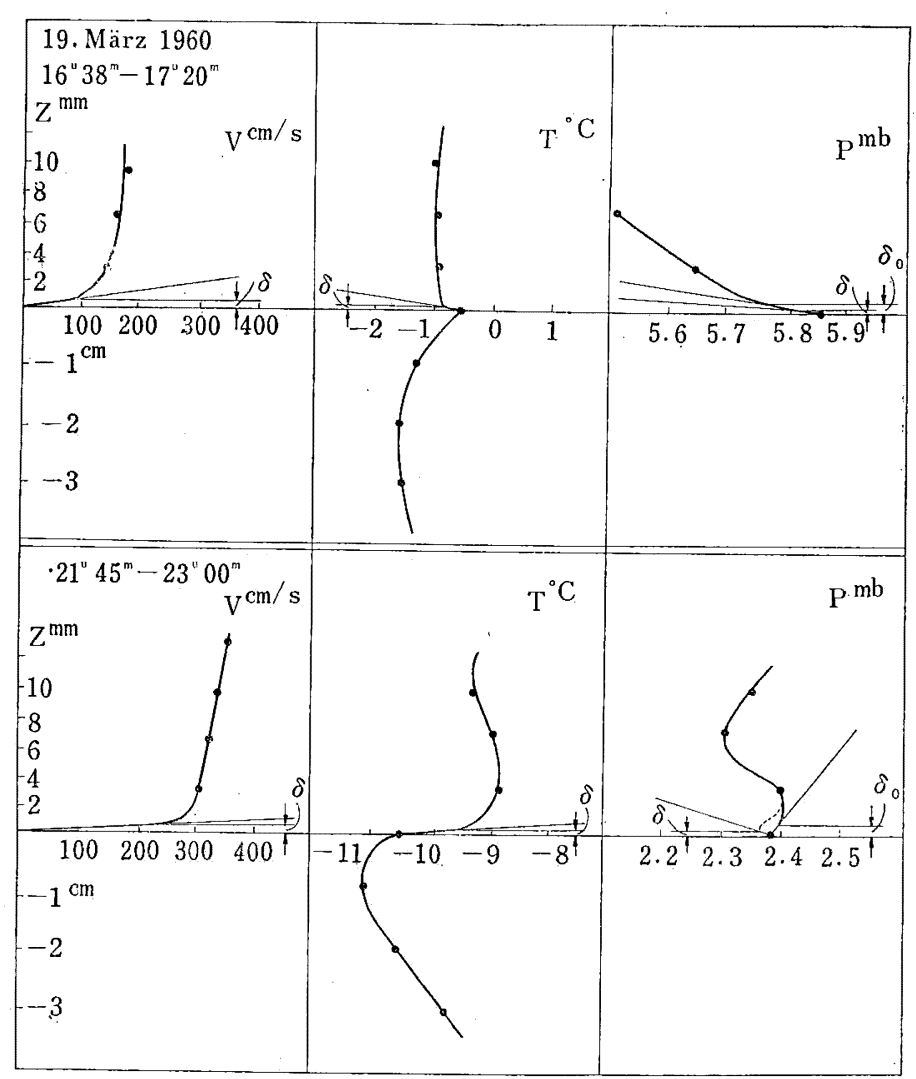

Abb. 6. Profile von V (Windgeschwindigkeit), T (Temperatur), $\mathrm{P}$ (Wasserdampfdruck) im Freien (Z: Höhe, $\delta, \delta_{0}$ : Tiefe der Laminarsubsicht).

itts, Abb. 6) wurden von den andern Forschern angeboten. Die Profile der Windgeschwindigkeit konnten mit den Ergebnissen des Experiments im Windtunnel im allgemeinen gegenübergestellt werden, wie es später gesagt wird.

Die Messungen der Temperatur und des Taupunktes waren frei im Sonnenschein auf einer Holzplatte durchgesetzt, aber die Aufgabe der Einwirkung des Sonnenscheins bleibt noch zurück, um gelöst zu werden. Die Höhe der Messung war von $0 \mathrm{zu} 3 \mathrm{~cm}$ und der Fluss des Wasserdampfs war, wie später gesagt,

$$
\begin{array}{ll}
\text { Tag } & -0.177 \mathrm{~g} / \mathrm{m}^{2} \min \\
\text { Nacht } & +0.004 \mathrm{~g} / \mathrm{m}^{2} \mathrm{~min} .
\end{array}
$$

\section{Diskussion}

(a) Vermutung der Tiefe der Laminarsubschicht durch das Experiment im Windtunnel

Vor der Forschung auf der Schneedecke ist das Experiment im Windtunnel nötig, um die Tiefe der Laminarsubschicht über der gegebenen Rauheit vermuten zu können. Eine 


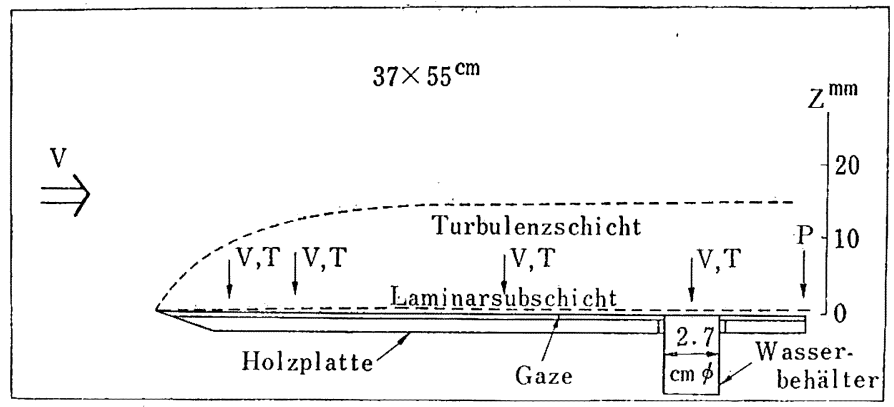

Abb. 7 Experiment im Wind tunnel.

dünne Holzplatte mit scharfen Enden wurde im Windtunnel gestellt (Abb. 7) und mit der immer mit Wasser angefeuchteten Gaze übergedeckt.

Die Verdamgfungsmasse wurde aus der Messung des Gewichtes des Wassers in einem kleinen in der Lee begrabenen Wasserbehälter bestimmt. In Wahrheit soll auch die Oberfläche des Wasserbehälters mit Gaze bedeckt werden, aber dann ist die Windgeschwindigkeit so klein, dass die Welle nicht gesehen wird, und es wird ziemlich gut mit dem Experiment von Yамамото (1949) übereinstimmen, dass $T$ (Oberfläche des Wassers) $=T$ (Oberfläche der Gaze).

Die Profile der Windgeschwindigkeit und Temperature wurden an den Distanzen 5, 10, $30,50 \mathrm{~cm}$ von dem Ende der Luvseite und die Temperatur der Oberfläche mit getauchten Thermistor gemessen, aber allein die Taupunkte wurden am Ende der Leeseite gemessen (Windgeschwindigkeiten waren $100 \mathrm{~cm} / \mathrm{s}$ und $280 \mathrm{~cm} / \mathrm{s}$. Die Höhe der Messung war möglichst fein, d. h. $0.5 \mathrm{~mm}$ od. $1 \mathrm{~mm}$ (Abb. 8). Unter $100 \mathrm{~cm} / \mathrm{s}$ auch wurden das Experiment gemacht und wenn die Profile in der Nähe der Oberfläche gerade Linien

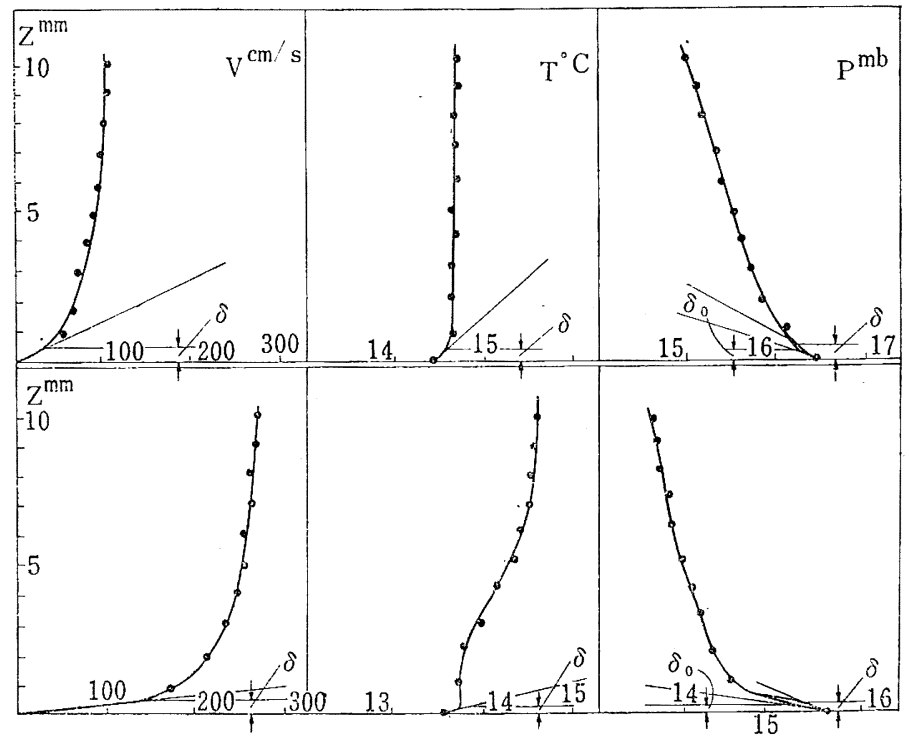

Abb. 8. Profile von V.T.P im Windtunnel $(x=50 \mathrm{~cm})$. 
darstellen, dann scheint uns vielleicht wahr, dass diese Gebiete die der Molekulardiffusion seien. Diese Hypothese wird aus dem Experiment und der Literatur nicht unrecht gedacht. In Abb. 7 werden die Turbulenzgrenzschicht und die Laminargrenzschichte eingeschrieben. Eigentlich waren viele Formeln fürs Turbulenzgebiet vorgelegt worden, aber sie sind fast alle das Logarithmen-Gesetz seit Prandtl (1938), oder Potenz-Gesetz, oder dessen Umformung wie die Formeln von Monin u. Obukhov (1954), oder nur die empirischen Formeln.

Wenn das Log.-Gesetz (1) auf unseren Date (das Experiment im Windtunnel und die Feldwerke) angewandt wird, kann die Differenz zwischen Theorie und Data wie in Tafel 1 dargestellt werden.

(1)

$\tau=\rho V_{*}^{2}=$ Konst.

$V=\frac{V_{*}}{\kappa} \ln \frac{Z}{Z_{o}}$

$T-T_{o}=T_{*} \ln \frac{Z}{Z_{o}}$

$Q-Q_{o}=Q_{*} \ln \frac{Z}{Z_{o}}$

$q=-\kappa V_{*} C_{p} \rho T_{*}$

$E=-\kappa V_{*} \rho Q_{*}\left(=\mathrm{W}_{\text {log. }}\right)$

wo $\begin{cases}\tau & \sim \text { Schubspannung in } \mathrm{g} / \mathrm{cm} \mathrm{sek}^{2} \\ \rho & \sim \text { Dichtigkeit der Luft in } \mathrm{g} / \mathrm{cm}^{3} \\ V & \sim \text { Windgeschwindigkeit in } \mathrm{cm} / \mathrm{s} \quad(=\mathrm{U}) \\ T & \sim \text { Temperatur }{ }^{o} \mathrm{~K} \\ Q & \sim \text { Spezifische Feuchtigkeit } \\ V_{*}, T_{*}, Q_{*} \sim \text { Friktion } V, T, Q \\ T_{o} & \sim \mathrm{T}\left(Z_{o}\right) \\ Q_{o} & \sim \mathrm{Q}\left(Z_{o}\right) \\ K & \sim \text { Kárman Konst. }(=0.43) \\ Z & \sim \text { Höhe in cm } \\ Z_{o} & \sim \text { Rauhigkeitsparameter } \\ q & \sim \text { Fluss der Wärmemasse in cal } / \mathrm{cm}^{2} \mathrm{sek} \\ C_{p} & \sim \text { Spezifische Wärme der Luft unter } \\ & \quad \text { Konstantdruck } \\ E & \sim \text { Fluss der Wasserdampfsmasse in } \mathrm{g} / \mathrm{cm}^{2} \\ & \text { sek }\end{cases}$

Tafel 1.

\begin{tabular}{|c|c|c|c|}
\hline Experiment & $\mathrm{U}$ & $100 \mathrm{~cm} / \mathrm{s}$ & $280 \mathrm{~cm} / \mathrm{s}$ \\
\hline & $\left|\left(\mathrm{W}-\mathrm{W}_{\mathrm{log}}\right) / \mathrm{W}\right|$ & $32 \%$ & $79 \%$ \\
\hline \multirow[t]{2}{*}{ Feld } & $U$ & $\sim 200 \mathrm{~cm} / \mathrm{s}$ & $\sim 400 \mathrm{~cm} / \mathrm{s}$ \\
\hline & $\left|\left(\mathrm{W}-\mathrm{W}_{\log }\right) / \mathrm{W}\right|$ & $17 \%$ & $>100 \%$ \\
\hline
\end{tabular}

Die Differenz vergrössert sich mit der Windgeschwidigkeit in dieser Tafel. Vielleicht wird der Fluss entlang der Vertikalrichtung nicht konstant sein, wenn sich die Windgeschwindigkeit vergrössert. Man muss auch das Experiment von YАMAмото in Betracht ziehen, aber wir wollen als eine direkte Methode, ins Molekulardiffusion-Gebiet eintreten. 
Die Formel des Molekulardiffusions im zylinderförmigen Wasserbehälter wird in (2) dargestellt.

$$
W=-\alpha D \frac{d p}{d Z} \pi a^{2} t
$$

wo

$$
\mathrm{W}(=\mathrm{M}) \sim \text { Verdampfungsmasse in } \mathrm{g} / \mathrm{cm}^{2} \text { sek }
$$

$$
\alpha \sim \frac{\rho_{o}}{p_{o}} \frac{T_{o}}{T}
$$

$\rho_{0} \sim$ Dichtigkeit des Wasserdampfs $\left(273^{\circ} \mathrm{K}\right)$ in $\mathrm{g} / \mathrm{cm}^{3}$

$T_{0} \sim 273^{\circ} \mathrm{K}$

$p \sim$ Wasserdampfdruck in $\mathrm{mb}$

$p_{0} \sim$ Gesättigter Wasserdampfdruck $\left(273^{\circ} \mathrm{K}\right)$ in $\mathrm{mb}$

$D \sim$ Diffusionskoeffizient des Wasserdampfs in Luft in $\mathrm{cm}^{2} / \mathrm{sek}$

$a \sim$ Radius des Behälters in $\mathrm{cm}$

$\mathrm{t} \sim$ Zeit in sek

$\frac{d p}{d Z}$ wird aus (2) berechnet:

$$
\mathrm{U} \mathrm{cm} / \mathrm{s} \quad \frac{d p}{d Z} \mathrm{mb} / \mathrm{cm}
$$

\begin{tabular}{rr}
\hline 100 & -8.07 \\
280 & -26.30 \\
\hline
\end{tabular}

Die Tiefe des Molekulardiffusion-Gebietes $\delta_{0}$ wird mittels des wirklichen Profils vermutet (als die Wassersättigung in der mittleren freien Weglänge) :

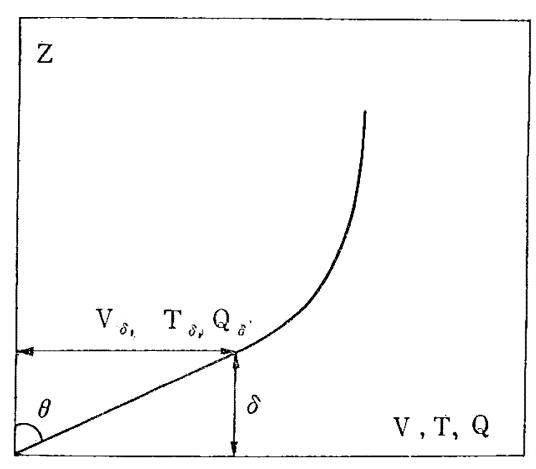

Abb. 9. Gradient von V,T,Q in der Nähe der Oberfläche.

\begin{tabular}{cc}
$\mathrm{U} \mathrm{cm} / \mathrm{s}$ & $\delta_{0} \mathrm{~mm}$ \\
\hline 100 & 0.4 \\
280 & 0.3 \\
\hline
\end{tabular}

Die Flusse der Windgeschwindigkeit und Temperatur wurden nicht gemessen, aber weil sie als Flüsse entlang der Vertikalrichtung sich nicht verändern, wurden ihre Gradienten in der Nähe der Oberffäche aus (3) und Abb. 9 mit Anwendung vom Log.-Gesetz im Turbulenzgebiet gerechnet. 
(3)

$$
\begin{aligned}
& \frac{\tau_{0}}{\eta}=\left(\frac{\partial V}{\partial Z}\right)_{0}=\frac{\rho V_{*}^{2}}{\eta}=\frac{V_{\delta}}{\delta_{v}}=\tan \theta_{v} \\
& \frac{q_{0}}{C_{p} D_{t}}=\frac{\kappa V_{*} T_{*}}{D_{t}}=\frac{T_{\delta}}{\delta_{t}}=\tan \theta_{t} \\
& \frac{E_{o}}{\rho D_{q}}=\frac{\kappa V_{*} T_{*}}{D_{q}}=\frac{Q_{q}}{\delta_{q}}=\tan \theta_{q},
\end{aligned}
$$

$$
\text { wo } \begin{cases}\tau_{0} & \sim \tau \text { auf der Oberfläche } \\ \eta & \sim \text { Molekularzähigkeitskoef. }=\rho D_{v} \mathrm{~g} / \mathrm{cm} \text { sek } \\ V_{\delta}, T_{\delta}, Q_{o} & \sim V, T, Q \text { auf der Laminarsubschicht } \\ \theta_{v}, \theta_{t}, \theta_{g} \sim \text { Winkel des Gradienten } \\ q_{0}, E_{0} \sim \text { q und E anf der Oberfläche } \\ D_{v}, D_{t}, D_{q} \sim \quad \text { Dif.-Koef. }\end{cases}
$$

Diese Gradienten $(\tan \theta)$ sind in Abb. 8 eingeschrieben und $\delta(V, T, Q)$ werden aus dem wirklichen Profil vermutet.

\begin{tabular}{cc}
$\mathrm{U} \mathrm{cm} / \mathrm{s}$ & $\delta \mathrm{mm}$ \\
\hline 100 & $0.3 \sim 0.6$ \\
280 & $0.2 \sim 0.4$ \\
\hline
\end{tabular}

So ist die Ordnung gleich $\grave{\delta}_{0}$. Auf der Wasseroberfläche wird $\delta$ von einer Vergleichung gerechnet.

Brauchen wir die Formel der Definition von Ogiwara (1944);

$$
\delta=\frac{1}{c_{0}-c_{\infty}} \int_{0}^{\infty}\left(c_{0}-c\right) d Z
$$

wo $\begin{aligned} & \left.\right|^{\delta} \sim \text { Tiefe des Molekulardiffusion-Gebietes auf der Wasseroberfläche } \\ & { }_{c} \sim \text { Dichtigkeit des Wasserdampfs }\end{aligned}$

$c_{0} \sim$ Dichtigkeit des Wasserdampfs auf der Oberfläche

$c_{\infty} \sim$ Dichtigkeit des Wasserdampfs ausserhalb der Grenzschicht

Wenn $U=100 \mathrm{~cm} / \mathrm{s}, x=50 \mathrm{~cm}$ (Distanz vom Ende abwärts),

$$
\delta=5.6 \mathrm{~mm}
$$

Und braucht man O Formel (1950):

$$
\delta=4.79 \sqrt{\frac{\nu x}{U}},
$$




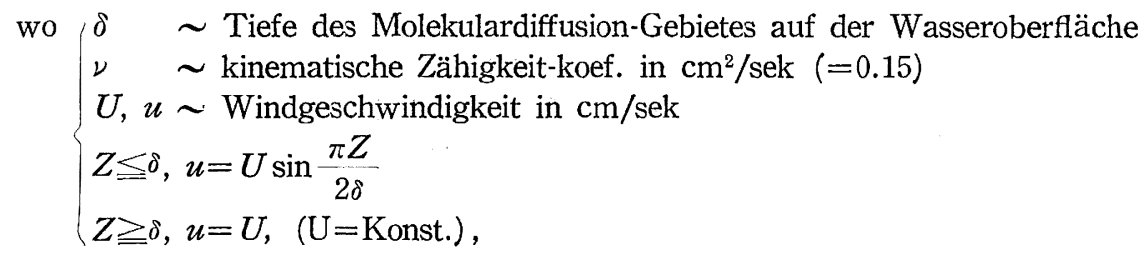

dann ergibt es sich, dass

\begin{tabular}{ccr}
$\mathrm{U} \mathrm{cm} / \mathrm{s}$ & $x \mathrm{~cm}$ & $\delta \mathrm{mm}$ \\
\hline 100 & 50 & 13.0 \\
280 & 50 & 7.8
\end{tabular}

Demnach ist es unverständlich, dass $\delta$ auf der Gaze nicht zu $\frac{\delta}{10}$ über dem Wasser der Subschicht entwickeln wird. Das Bild $\frac{x}{\delta}$ gegen $\operatorname{Re}=\frac{U x}{\nu}$ ist in Abb. 10 dargestellt. Über dem Wasser $\frac{x}{\delta}=0.209(\mathrm{Re})^{0.52}$ (Oshidas Formel), und über die Gaze $\frac{x}{\delta}=$ $0.00386(\mathrm{Re})^{1 \cdot 19}$ (Experimentische Formel), aber aus dem Experiment Uedas (1956), $\frac{x}{\delta} \rightarrow$ Konst. $(\delta=0.75 \mathrm{~cm})$ wenn $U \rightarrow 0$.

Glatter als die Gaze, das ergibt eine Annäherung nach der unteren Linie und rauher als die Gaze, das ergibt das Entfernen von der oberen Linie. (Das Beitragen des $x$ ist nicht so wichtig, denn wirklich nicht vergrössert $\delta$ auf der Gaze mit $x$, aber die Grenze zwischen $\frac{x}{\delta}$ im Laminargebiet und $\frac{x}{\delta}$ im Turbulenzgebiet $\left(\operatorname{Re} \approx 10^{4}\right.$ von Yamamotos Experiment) ist noch nicht klar.

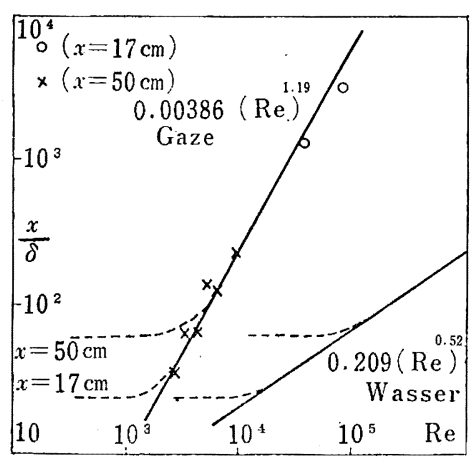

Abb. 10. Verhältnis von $\frac{x}{\delta} \quad$ zu $\quad \operatorname{Re}=\frac{U x}{\nu}$
Durch diese Anfangsforschung können $\delta$ und damit der Fluss auf einer oberfläche mit der Rauhigkeit der Gaze (vielleicht ist es gleich die der Schneedecke) und im Fall von $V<$ mehrere hundert $\mathrm{cm} / \mathrm{s}$ und $T>$ etwa $-10^{\circ} \mathrm{C}$ in gewissem Grad der Genauigkeit gerechnet werden.

Und in Zukunft, wenn eine feinere Taupunktmessung gemacht wird, wird $\delta$ obwohl in stärkeren Windgeschwindigkeiten mit grösserer Genauigkeit gemessen werden können.

Dann wird man $\frac{d p}{d Z}$ in der Laminarsubschicht im Zussamenhang mit $\frac{d p}{d Z}$ in der Turbulenzschicht vermuten können.

(b) Vermutung der Tiefe der Laminarsubschicht auf der Oberfläche der Schneedecke

Der Fluss wird auch in diesem Falle wie im Falle des Experiments in Windtunnel 
gerechnet.

Aber der Beitrag des Flusses von der inneren Schneedecke muss aus dem ganzen Flusse abgezogen werden. Im Experiment von Yoshida (1950) ist das Dif.-Koef., in der Schneedecke $\mathrm{D}_{s}$ etwa 0.8 (wenn die Dichtigkeit der Schneedecke 0.15 0.25 ist).

So ist der Fluss in der Schneedecke $W^{\prime}$ wie folgt:

$$
\begin{aligned}
& \begin{aligned}
\mathrm{W}^{\prime} & =-\alpha^{\prime} \mathrm{D}_{s} \frac{d T}{d Z} \pi a^{2} t \\
& =-\frac{(2.2 \sim 2.3) \times 10^{-4}}{L_{s}} \frac{d T}{d Z} \pi a^{2} t,
\end{aligned} \\
& \text { wo }\left\{\begin{array}{l}
\alpha^{\prime} \sim \frac{\rho_{0} T_{0}}{p_{0} T} \\
L_{s} \sim \text { Sublimationswärme }
\end{array}\right.
\end{aligned}
$$

$\delta_{0}$ wird aus (2) gerechnet, nämlich $\sim 0.5 \mathrm{~mm}$ und $\delta \sim(0.2 \sim 0.3) \mathrm{mm}$. So bleibt die Ordnung nicht verändert (als die Eissättigung auf der Oberfläche), d.h. die Oberfläche der Schneedecke kann sich der Oberfläche der Gaze sehr nähern. Und wenn der Zusammenhang zwischen $\delta_{0}$ und $V$ klar ist, wird der Fluss mit gewisser Genauigkeit aus der gegenwärtigen Beobachtung gerechent werden.

Man muss darauf anfmerksam sein, dass die Bestimmung der Temperatur auf der Oberfläche der Schenndecke sehr schwer ist, und dass die Einwirkung des Sonnenscheins unvermeidlich ist. Gewöhnlich ist der Subschicht schärfer als in der Turbulenzschicht, und wenn $\frac{d P}{d Z}$ vergleichsweise gross ist, der Fluss sich nicht so verändert obwohl $\mathrm{p}$ mit dem kleinem Fehler.

Zum Beispiel, wenn das Taupunkt als um $0.1^{\circ} \mathrm{C}$ höher (od. niedriger) gerechnen wird, sind die Differenz des Flusses

\begin{tabular}{cc}
$\mathrm{U} \mathrm{cm} / \mathrm{s}$ & $\frac{W-W_{0.1^{\circ} \mathrm{C}}}{W}$ \\
\hline 100 & $12 \%$ \\
280 & $4 \%$
\end{tabular}

Also wird die Genauigkeit des Flusses besser sein.

\section{Metamorphose der Kristalformen in der Schneedecke durch den Fluss des Wasserdampfs}

Die Eiskristale des Schnees fangen an, sich gleich nach dem Fallen auf der Schneedecke umzuformen. Wenn auch die Temperatur in der Schneedecke unter $\mathrm{O}^{\circ} \mathrm{C}$ ist, entsteht der Fluss des Wasserdampfs, wegen der Änderung des Sättigungswasserdampfdrucks mit dem Gradient der Temperatur.

Die mikroscopischen Photographien von Replicas (Lösung von $2 \%$ Vinyl-Formal) welche am Morgen um 7 Uhr gesammelt wurden, sind in Photos $4 \sim 10$ dargestellet.

Auf der Oberfläche Photos $4 \sim 7$ erscheinen die Plateauformen am meisten $(56 \%)$, dann die Plateauformen mit Zweigen (41\%), und am wenigsten die Dendriten und unklare Formen. Die Längendurchschnitt von $0 \sim 5 \mathrm{~cm}$ Tiefe (Photo $8 \sim 10)$ zeigen Plateau (40\%), Dendrit $(40 \%)$, Plateau mit Zweigen (21\%). In grösserer Tiefe fängt die Eiskristalform die sich umzuformung, aber die Kristalle sind noch individual und die Eisbrücken oft bemerkbar. Einige unter den Dendritenformen haben ihre nur oberflächliche Umformung wie in Photo 10. Das ist der Fall, wo der Wasserdampf nur nach einer Richtung, sage 


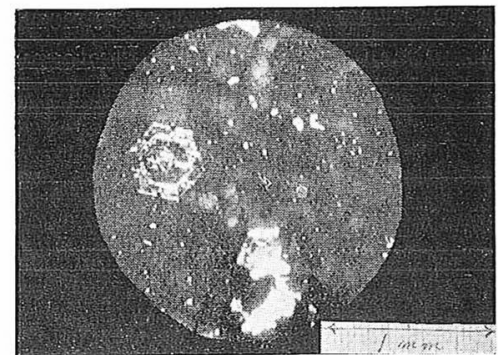

Photo 4. Replica auf der Oberfläche, $7 \mathrm{Uhr}$.

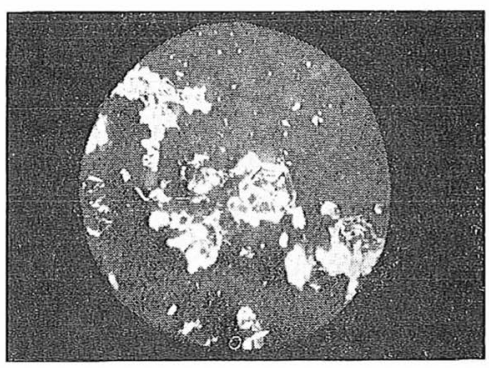

Photo 5. Wie oben.

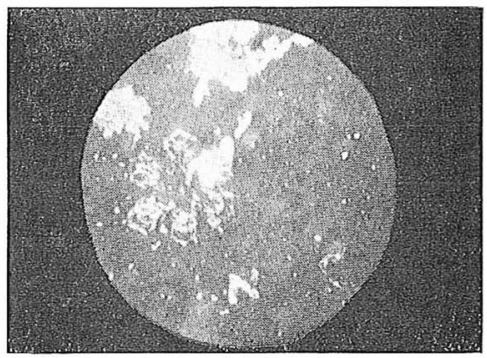

Photo 6. Wie oben.

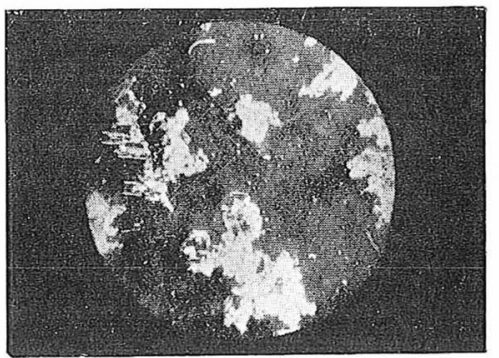

Photo 7. Wie oben.

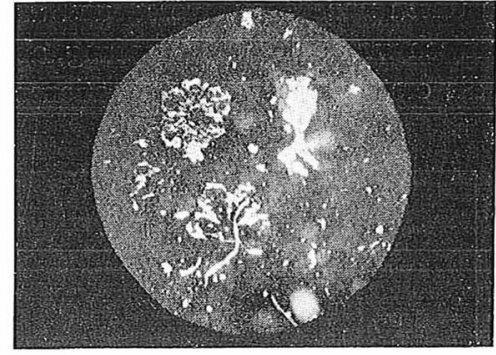

Photo 8. Längendurchschnitt (0 5 cm), 7 Uhr.

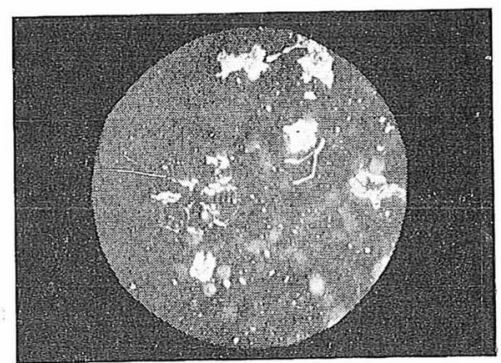

Photo 9. Wie oben.

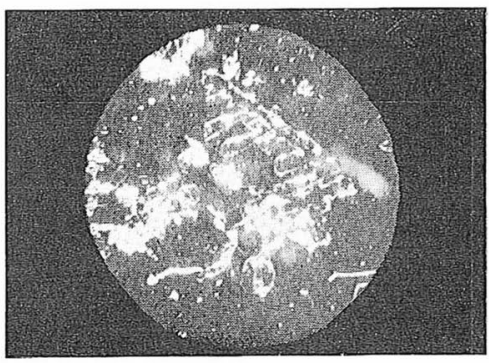

Photo 10 . Wie oben.

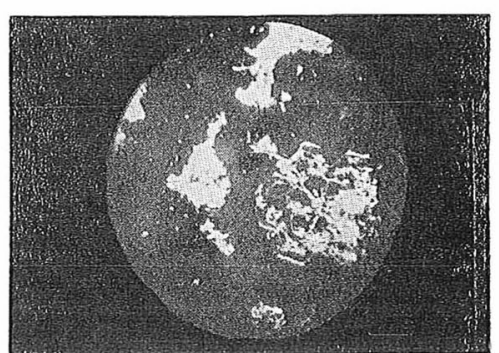

Photo 11. Auf der Oberfläche," $18 \mathrm{Uhr}$. 
von unten nach oben, fliesst, und die Kristalle sind unten kristallinisch und oben nonkristallinisch wegen der Verdampfung, aber dies ist nicht statistisch erklärt. Diese Umformung ist die erste Reihe von Sublimationsmetamorphose.

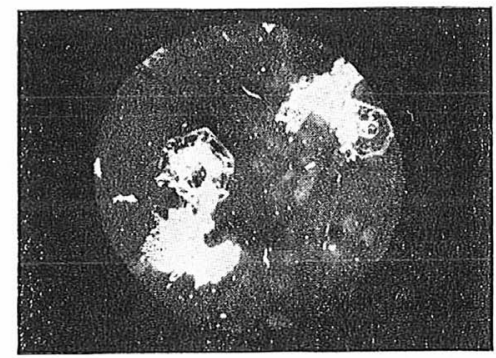

Photo 12. Wie oben.

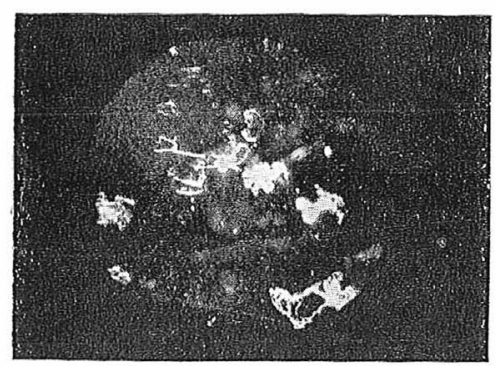

Photo 13. Wie oben.

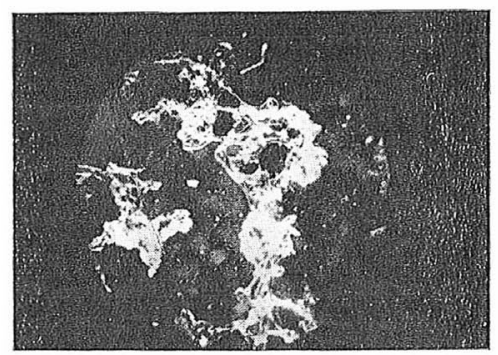

Photo 14. Längendurchschnitt $(0 \sim 5 \mathrm{~cm}), 18$ Uhr.

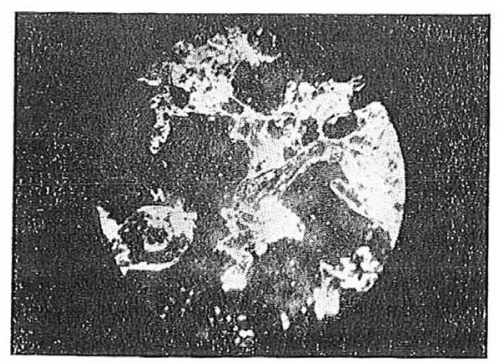

Photo 15. Wie oben. ๆ!

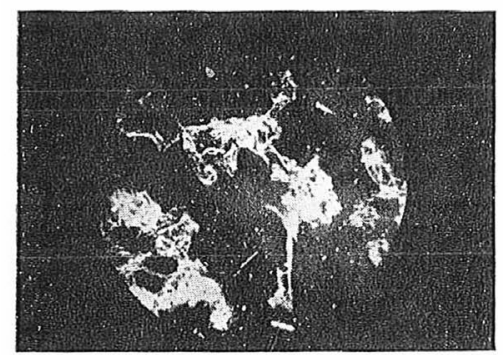

Photo 16 . Wie oben.

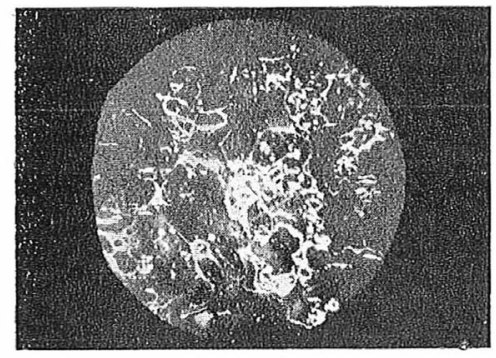

Pho๖o 17. Läng endurchschnitt (15 2) $\mathrm{cm}), 18$ Uhr.

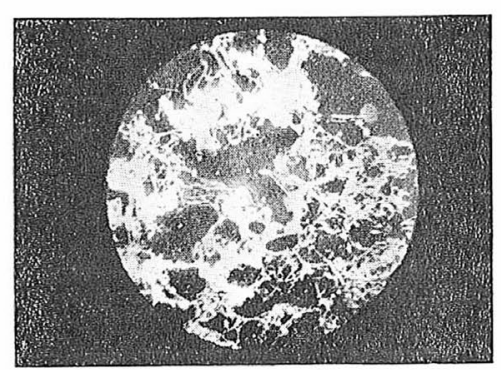

Photo 18. Wie oben.

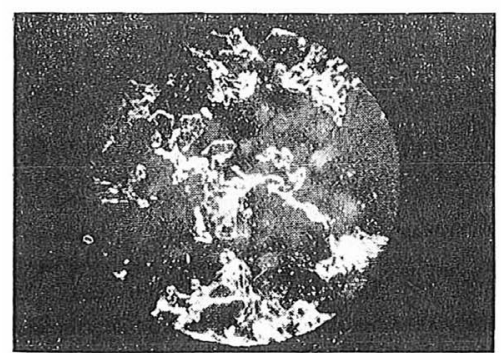

Photo 19. Wie ole.1. 
Am Abend um 18 Uhr wird die Zahl der Kristalle sehr wenig (Verminderung von einer Stelle) und die Ecken sind rundlich (Photos 11 13). Der Oberflächenreif hat sich schon in einigen Kristallen entwickelt (Photo 12).

Die Längendurchschnitte von $0 \sim 5 \mathrm{~cm}$ sind in Photos $14 \sim 16$ gezeigt. Trotzdem das Temperaturgradient nur $0.27^{\circ} \mathrm{C} / \mathrm{cm}\left(0 \mathrm{~cm} \sim-7.2^{\circ} \mathrm{C},-10 \mathrm{~cm} \sim-4.5^{\circ} \mathrm{C}\right)$ war, war die Umformung so schwer, dass die Originalformen kaum erkannt wurden. Dies war nur durch den Fluss des Wasserdampfs erzeugt worden, weil die Temperaturen unter $\mathrm{O}^{\circ} \mathrm{C}$ waren (Yoshida 1950). Es hatte gereift durch den Fluss von oben nach unten auf der Oberfläche, (deren Temperatur durch die Radiation unter den Taupunkt fiel.)

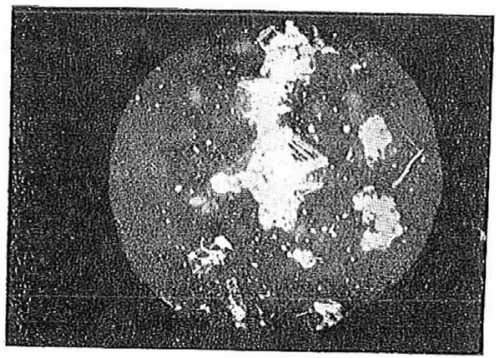

Photo 20. Auf der Oberiläche, 23 Uhr.

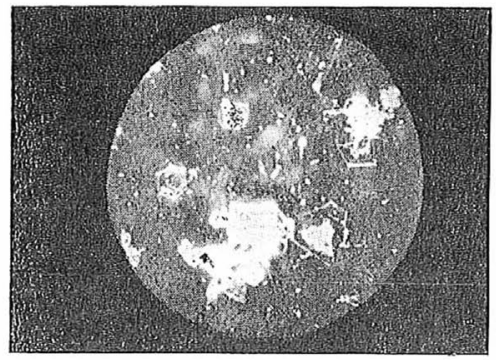

Photo 21. Wie oben.

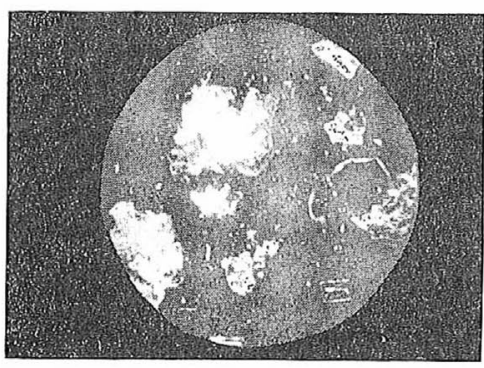

Photo 22. Wie oben.

Die Eisbrücken waren bemerkbar. Es ist die zweite oder die dritte Reihe von Sublimationsmetamorphose.

In den Längendurchschnitten von $15 \sim 20 \mathrm{~cm}$ (Photos 17 19) war die Umformung noch viel schwerer (vielleicht von noch stärkerem Temperaturgradienten), und es gibt kaum die Eisgrosskorm Die Eisbrücken kamen durcheinander und es ist die dritte Reihe von Sublinationsmetamorphose.

Mitternachts (23 Uhr) war nur der Oberflächenreif gesehen, der sich von den Grosskörnen auf der Oberfläche entwickelt hatte, und es gab kaum andere Kristallformen (Photo 20 22). Wir bemerken die Vortrefflichkeit der Sublimation des Wasserdampfs. Noch dazu wollen wir eine Erscheinung um Mitternacht zusetzen, d. h. der Schnee in der Oberfläche war der Rauhschnee, aber in der Tiefe von $0.5 \sim 1.0 \mathrm{~cm}$ gab es den vergleichsweise festen Rauhschnee (einige $\mathrm{cm}$ dick). Und noch unten, da war der Rauhschnee wie der Oberfäche.

Das Gebiet der minimalen Temperatur war $1 \sim 2 \mathrm{~cm}$ unter der Oberfläche, und dies Gebiet war von der Sublimation des Wasserdampfs entstanden, da es nahe dem tiefen Reif war.

Nach SaIto (1945), im allgemeinen erscheint die erste Reihe nach 1 5 Tagen, die zweite Reihe nach 1 3 Wochen, und die dritte Reihe nach 4 10 Wochen, aber dennoch haben wir gefunden, dass nach nur einem Tage die zweite oder dritte Reihe von Sublimationsmetamorphose in der Oberfläche mit dem starken Temperaturgradient entstand.

\section{Schlussbemerkung}

(a) Im gegenwärtigen Zustande, wenn die Windgeschwindigkeit unter einige hundert $\mathrm{cm} / \mathrm{s}$ und die Temperatur über etwa $-15^{\circ} \mathrm{C}$ ist, kann die Tiefe der Laminarsubschicht auf einer gewissen Oberfläche (z. B. feuchte Gaze od. Schneedecke) von der Taupunktmessungen (in 
der vergleichlich rechte Genauigkeit) und vom Flusse des Wasserdampfs bemessen werden. (b) Diese 'Tiefe ist ziemlich dünn, d. h. in der Ordnung von etwa $0.4 \mathrm{~mm}$ in $U=100 \mathrm{~cm} / \mathrm{s}$, and wird mit der zunehmenden Windgeschwindigkeit schnell dünner von etwa $7.5 \mathrm{~mm}$ in $\mathrm{U}=$ null $\mathrm{cm} / \mathrm{s}$.

(c) Es scheint mehr einfacher und unmitterbarer in Prinzip und vergleichlichsweise genauer; wenn der Fluss von dem Gradient des Taupunktes in der Laminarsubschicht statt in der Turbulenzschicht gerechnet werden könne.

(d) Von den Replica-Microphotograhien in den Oberflächengebieten der Schneedecke gesehen, ist die Umformung schwerer in der inneren Tiefe unter $\mathrm{O}^{\circ} \mathrm{C}$ wegen der Sublimation des Wasserdampfs. Die Geschwindigkeit der Metamorphose ist ziemlich schnell durch das starken Temperaturgradient, und nach einem Tag kommt die dritte Reihe von Sublimationsmetamorphose $(20 \mathrm{~cm}$ Tiefe), und dann können kaum die Grosskörne geformt werden, noch die Eisbrücken entwickeln. Nachts entwickelt der Oberflächenreif.

Dankbarkeit:- Der Verfasser ist dem Herrn Dr. TAKAHAshi (Met. Res. Inst.) für seine Leitung bei der Beobachtung und dem Experiment und den Herren Soma, Nemoto und SAsho (Met. Res. Inst.) für ihre Hilfe und Eingebungen zum tiefen Dank verpflichtet.

\section{Appendix 1}

In der letzten Literatur forschte Ueda (1960) über der Verdampfung des Wassers von der Zwangskonvektion weiter.

Aber werden die folgenden Fragen aufgemerkt.

1. Der elektrische Ventilator wurde anstatt des Windtunnels gebraucht.

2. Die Verdampfungsgeschwindigkeit wurde nicht gemessen unmitterbar.

3. Die Temperatur der Verdampfungesoberfläche und die Profile der Windgeschwindigkeit und der Temperatur auch nicht gemessen werden. (Jeder $\delta$ wird in dem gleichartigen Ordnung übereinstimmen.)

4. Der gerade Gradient des Wasserdampfdruckes wird auch in dem Transitionsgebiet beobachtet werden können.

5. $x$ und $\delta$ werden vielleicht verändert werden, (denn $x$ in dem Laminarfluss im Windtunnel wird nicht gleich $\mathrm{zu} x$ in dem Turbulenzfluss von dem Ventilator und $\delta$ wird vielleicht verkleinert werden), so der Beitrag wird einwirken auf das Bild $\frac{x}{\delta}$ gegen Re.

\section{Appendix 2}

Die Beobachtungen von Martinelli (1960) an Alpine Schneefeld (Zentral Colorado) im 1957 und 1958 sind mit dieser Forschungen in der gleichen Neigung wie folgt.

1. Die täglich Neigung des Wasserdampfaustausch ist Verdampfung im Morgen, Kondensation im Nacht, und jeder Zustand im Nachmittage infolge des Wetterzustandes.

2. Die Flüsse des Wasserdampfs (In der Kondensation die mittere Austauchkoeffizient ist $+0.0263^{\prime \prime} /$ Tag und in der Verdampfung -0.0268" /Tag, mit dem ungefähr gleichgrössen Gefäss $\left.\left(16^{\prime \prime} \phi\right)\right)$ zeigen mit dieser Forschungen gute übereinstimmung. 


\section{Literaturen}

Aокт, M., 1957: Recent Progress in Thermoelectric Refrigeration. J. Appl. Phys., Japan, 26, No. 6, 215.

AokI, M., 1959: Thermoelectric Cooling by Bi-Te Thermojunctions, ibid., 28, No. 2, 77.

Corcoran, W.H., Opfeli, J.B., and Sage, B.H., 1956: Momentum Transfer in Fluids. Acad. Press, Inc. Pub., New York.

Monin, A.S. and Oвикног, A. M., 1954: Fundamental Law of the Turbulent Transport in the Atmospheric Surface Layer. Akda. Nauk. SSSR, Trud. Geofiz. Inst., No. 24 (151), 1963.

Marinemi, M. Jr., 1960: Moisture exchange between the atmosphere and Alpine snow surfaces under summer conditions (Preliminary results) J.M. 17, No. 2, p.227 231.

OGiwara, S., 1944: On the Evaporation of Water from a Vessel of Finite Dimension in the Case of Laminar Boundary Layer (Studies on Evaporation, Part 1). Met. Soc. Jap. 22, No. 4, 127.

OshidA, I., 1950: (Im Japanischen) Verdampfung und Austrocknung. Kawaide Shobo.

SaIto, R,, 1945: (Im Japanischen) Strukture der Schneekörner und ihre mechanischen Eigenschaften. J. Met. Soc. Jap. 23, No. $1 \sim 12$.

Satro, R., 1949: Physics of Fallen Snow. Geophys. Mag. (C.M.O., Tokyo) 19, Nos. 1 2.

Soma, K., Uchida, E. and Nemoto, S., 1958: On the heat budget in the surface layer of the snow cover (1). Reported in Met. Congress, May.

UEDA, M., 1956: Measurements of the Gradient of Water-vapour Pressure and the Diffusion Coeffcient. J. Appl. Phys., Japan, 25, No 4, 144.

UedA, M., 1960: Rate of Evaporation of Water by Forced Convection. J. Appl. Phys. Jap., 29, No. 7, p. $443 \sim 451$.

UCHida, E., Nenото, S., and Soma, K., 1959: On the heat budget in the surfaee layer of the snow cover (2). Reported in Met. Congress, May.

Yanamoto, G., 1949: Some Experiments on Evaporation from Finite Surfaces. J. Met. Soc. Jap. 27, No. $10,318$.

Yoshida, Z. and Kunorwa, D., 1950: Sublimation in the Interior of Snow Layer. Low Temperature Science, 3, 89.

Yoshidd, Z. and Iwat, Y., 1950: Measurement of Thermal Conductivity of a Mass of Snow. ibid., $3,79$.

Yoshida, Z. and Kozima, K., 1950: Metamorphosis of snow Crystals, ibid. 5, 75.

Yoshida, Z., 1950: Heat Transfer by Water Diffusion in the Interior of Snow Layer. ibid, 5, 93. 


\title{
積雪面に於ける水蒸気の拡散
}

\author{
内田英 治
}

積雪面よりの蒸発量を求める為に 従来種々の公式が乱流領域にて導かれているが 若し風速が比較 的小さく 粗度が或程度以上大きくないならば 境界面上の分子拡散領域の厚さが推定出来 それによって 水蒸気勾配が計算されるので 蒸発量注求めることが出来るこの方が原理が簡単で精度るよいと思われ るので 積雪面上で (文実験室内で) 風速 温度 湿度のプロフィルを測定してこの厚さを推定して見た 野外に於いては同時に積雪表層の雪のレプリカを採取し 顕微鏡により粒子の変形を調べた 